\title{
Testículo no palpable. Características y tratamiento
}

\author{
José Manuel Escala A.'; Graham Smith²; Howard Snyder ${ }^{2}$; John Duckett ${ }^{2}$
}

\begin{abstract}
Resumen
Otjeltvo: describur los resuliados del manejo quirúrgico abierto en pacienles con teslículos no descendidos pocientes y métodcs se registrcton las caracteristicas macroscópicas de los tesles, su ubicación y los resultados oxeratcrios er 1800 niños con testiculos no descendidos, operados en un centro estadounidense entre 1986 y 1094. Resuritodos: en 447 casos los testículos eran no palpables, en $58 \%$ el izquerdo, el derecho en $35 \%$ o ambos en $7 \%$. En la operación no se erconlraron testículos o eran aróficos en $: 81$ [4 1\%) pacientes, en 136 (30\%) estoban en e canal 'ngunal, en 92 (2) $1 \%$ ) estoban en el interior del abdomen y en 38 |8\% se ubicaban en olros lugares. En $3 E$ de los 92 poc entes con testes irtraabdominales se usó el método operatorio de Fowler-Stephens, que dio buen resulado en 28: en otros 33 se usó la disección intraperitoreal y movilización del testículo sin sección de vascs espermáticos. con buen éxilo en 31 cosos; en $b$ se usilizo el procedimiento en dos elopos, logranóc llevar el leste al essrcto en cinco. En ? 5 pacertes fue necesaria la orquiectomía, por atrofia lesticular, que, en 10 de ellos, se acompcñobo de disocioción epididimo testic ulor completa. Conclusión sólo una fracción relotivarsente pequeña de les pacienles, elyos tesles son de locolizac én intrabadominal, se podrían habe beneficiado con el diagnístics laparosccpico. Lo exploracion inguinal con extens ćn abdominal siempre fue suficiente para localizar el lesticulo. vascs o deferente cuanoc el testículo estabo ausente. Eri la mayoría ce los casos de lestículos no palpables no atrólicos, se logrc descencerlos satistactoriamente al escroto
\end{abstract}

[Palabras clave: testículos no descendidas, criptorquidos.]

\section{Undescended non palpable testis}

Objoctive 10 describe results of open surgicai manogement in non palpable testis in a large series of patients. Potients and methods. Eelween january 1986 ard june 1994. 1 B6o boys with undescended lestes were trealed. 447 of their lestes were no valpable of presentotion. Average age of patients al presentation was 34 mon'hs. Surgical managerrent consisted in etther Fowler. Slepheris or standard orchicosexy fwith out dividing the spermatic vessels respectively). Results: at surgery location was idertified ir. 260 lestes wereas $181 \quad(40 \%$, testes were not found or were extremely atroshic. Ninelywo $(2 ; \%$ t anong identified tesles were intra-abdomincl, 136 130\%) were located

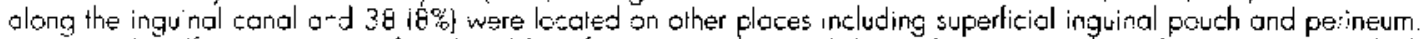
Asscoiated malformations were found in $39 \%$ of patients with inlroabdominal testes, mainly in the urinary tract which included eight cases of hypospadias. The aHachment of the epididyrnis to the testis was abnormat in 23 out of 54 $136 \%$ ) cases in which this warecorded, 10 being competely unattached cnd 13 partially attached 10 epididymal head or tol. Processus vaginalis was widely palent in $88 \%$ iniraobdominal coses, sometimes sliding into the canal. Conctisions: only c reduced number of paitenls intrajódominal testes might have benefit irom. diagnostic lopcroscopy for ocalization of their estes wrile the semander woud still require open surgical inguinal exploration. Results look sliglitly better wilt slandaid orchidopexy with ou dividing line spermatic vessels tan with Ine. Fowier-Slephens method.

(Key words: undescended lestis.i

El escroto vacío es uno de los motivos más frecuentes de consulta en urología pediátrica.

I. Servicio de Urología. Clínica Las Condes. Santiago, Chile.

2. Deparlamento de Urología. Hospital de Niños de Filadelfia. Filadelfia. EUA.
La criptorquidia es la causa más común y puede afectar a aproximadamente 1 de cada $150 \mathrm{ni}$ ños $^{1}$, siendo, en la mayoría de los casos testículos incompletamente descendidos, localizados en cualquier parte entre el abdomen y la raíz escrotal. En una proporción también considerable de pacientes, cercana a $20 \%$, no es posible 
percibir el testículo por palpación ${ }^{2-7}$. Este análisis se refiere al testículo no palpable.

La laparoscopía ha sido utilizada con cada vez mayor frecuencia desde 1976 para ubicar testículos no palpables ${ }^{9}$. Posteriormente se la empleó en la primera etapa de la técnica de Fowler-Stephens en testículos intraabdominales ${ }^{10}$, para la orquiectomía cuando es necesaria en algunos pacientes ${ }^{11.12}$ e incluso en la segunda etapa de Fowler-Stephens ${ }^{13,14}$.

En e] Departamento de Urología del Hospital de Niños de Filadelfia no se usan de rutina laparoscopía, ultrasonografía, tomografía, angiografía venosa o resonancia nuclear para ubicar testículos no palpables, por la alta probabilidad de falsos positivos o negativos. Se prefiere, en cambio, la exploración quirúrgica como método único para confirmar el diagnóstico $\mathrm{y}$ hacer el tratamiento.

Con el propósito de ilustrar el rendimiento de esta forma de proceder se describen las características de los testículos no palpables, su ubicación y los resultados del tratamiento.

\section{Material y Método}

Desde 1986 en el Departamento de Urologia del Hospital de Niños de Filadelfia se registran todos los pacientes con testículo no descendido en un protocoto especialmente diseñado para su estudio posterior. Se anotaron edad del paciente, posición del testículo afectado antes de la operaciór, estado del testículo contralateral y malformaciones asociadas, la ubicación confirmada en la operación y las características macroscópicas del testículo (tamaño, persis. tencia de conducto peritoneo-vaginal y relación entre epidídimo y deferentes).

Durante el periodo comprendido entre enero de 1986 y diciembre de 1994, se intervino quirúrgicamente en 1866 pacientes por testículo no descendido. En 447 casos los testículos no eran palpables en el examen preoperatorio $y$ constituyen el material de este anślisis. En 92 casos los testículos eran intraabdominales y en ellos se analizaron las características anatómicas. El tratamiento quirúrgico de los testículos íntrabdoninales se realizo empleando ya sea el procedimiento de Fowler-Stephens (sección de los vasos espermáticos para descender el teste, con preservación de vascularización de vecindad y deferencial, que se realiza en uno o dos (iempos) o la orquidopexia estándar, sir sección de vasos espermáticos. El resultado del tratamiento fue calificado como bueno si la posición y tamano finales testiculares fueron completamente satisfactorios; regular si la posición fue satisfactoria, pero había alguna disminución de iamar̃o de la glándula o si el tamajo de esta era satisfactorio pero la posición intraescrotal era alta: malo si ocurrió atrofia con disminución importante de tameño o desaparición del testículo.

\section{Resultados}

Los 447 testiculos no palpables representan $24 \%$ del total de testículos no descendidos atendidos en nuestro departamento. La edad promedio de los niños al ser intervenidos era $34 \mathrm{me}-$ ses, $63 \%$ menores que dos años. En 55\% de los casos el testículo no palpable era el izquierdo, en $31 \%$ el derecho y en $7 \%$ ambos.

La localización del teste identificada en el acto quirúrgico, antes de movilizar la glándula se describe en la tabla. En altos porcentajes los testículos eran atroficos, no detectables $(40,5 \%)$ o canaliculares $(30,4 \%)$. Sólo $92(22,5 \%)$ eran verdaderamente intraabdominales. Entre estos últimos las malformaciones asociadas fueron más frecuentes en los casos bilaterales y consistían en abdomen en pruna ( $n=5$ ), hipospadias $(n=8)$, otras anomalías urogenitales $(n=9)$, cardiovasculares $(\mathbf{n}=3)$, gastrointestinales $(\mathbf{n}=$ 4) y de otros aparatos y sistemas $(n=4)$.

La permeabilidad del conducto peritoneovaginal fue analizada en 73 de 92 casos de localización intraabdominal, siendo permeable en 50 de ellos, 25 a cada lado. La relación entre el epidídimo y el conducto deferente fue estudiada en 64 pacientes, siendo normal en 40 (total en 27 , en asa en 13) y anormal en 23. De los casos con relación anómala, en 10 pacientes había disyunción completa entre epidídimo y deferente (en nueve de ellos se extirpó el teste, que en general era pequeño y muy altos); en 1 l casos la unión se producía sólo en la cabeza del epidídimo, en dos sólo en la cola y en un caso no había deferente.

\section{Tabla}

Localización intraoperatoria de los testículos no palpables en el examen clínico

\begin{tabular}{lrr}
\hline & $\mathrm{n}$ & $\%$ \\
\hline Intraabdominal & 92 & 27.5 \\
Canalicular & 136 & 20.4 \\
Anillo & 22 & \\
Raíz escrotal & 2 & 8.5 \\
Bolsillo inquinal superficial & 12 & \\
Perigeal & 2 & \\
Atrofico o ausente & 181 & 40.5 \\
Total & 447 & \\
\hline
\end{tabular}


Respecto al tratamiento quirúrgico, hasta 1990 se usaba de preferencia el método de Fowler-Stephens, que se cmpleó en 39 casos de esta serie con buenos resultados en 23, regulares en 5 y malos en 10 con testes atróficas $(26 \%)$. A partir de esa techa. se ha preferido la orquidopexia estándar sin seccionar los vasos espermáticos, que se empleó en 33 pacientes en los que sôlo se han registrado dos testes atróficos. En anbos períodos el procedimiento en dos tiempos sólo se usó en scis casos. La orquectomía se realizó en 14 pacientes: en nueve con disyunción completa entrc epidídimo y deferente, en uno sin deferente y cn cuatro con teste afectado muy pequeño y alto asociados a teste contralateral normal. En síntesis, el resultado operatorio fue bueno en $79 \%$ de las orquidopexias estándar $y$ en $60 \%$ de intervenidos por método de Fowler-Stephens. En cinco casos de cada tipo de procedimiento el resultado fue regular, con un teste de buen tamaño, sin atrofia pero de ubicación final algo alta.

\section{Comentario}

En caso de leslículos no descendidos es de gran interés saber. antes de la operación. que este puede ser atrólico o no existir. Io que puede ocurrir en más de dos tercios de los casos ${ }^{15 .}{ }^{16}$. La pusibilidad de encontrar ef testículo en el interior del abdomen también es importante. ya que estos pacientes podrían beneficiarse con laparoscopín, especialmente si son bilaterales, aunque representan tan sólo una mínima proporción en nuesira serie de testículos no palpables.

El alto número de testículos en posición canalicular (un tercio del total de testículos no palpables) guarda probablemente relación la gran incidencia (casi 70\%) de permcabilidad peritoneo-vaginal, que en la mayoría de estos casos cra amplia. Estos testículos podrian haber sido palpados en el examen preoperatorio en el canal inguinal empleando presión positiva intraabdominal, pero aun así en todas las series no se logra derectar proporciones variables de ellos, entre $29 \%$ y $80 \%{ }^{17-19}$. La permeabilidad persistente del conducto peritoneo-vaginal tiene gran importancia para la viabilidad del testículo, ya que cuando ello no ocure los testículos faltan o son en extremo atróficos ${ }^{16}$.
Los resultados operatorios parecen mejores en casos de testículo intraabdominal con orquidopexia estándar empleando un pedículo ancho de peritoneo, para evitar la devascularización propia de las grandes disecciones ${ }^{20}$, que con el método de Fowler-Stephens. Los casos con atrofia testicular resultante entre estos últimos son los que evidentemente se habrian beneficiado con la laparoscopía, al instalar un "clip" en vasos espermáticos como primer tiempo, para más tarde descender el testiculo en un segundo tiempo, con o sin laparoscopía. La exploración inguinal con prolongación intraabdominal ha sido exitosa en localizar el teste, el deferente, o los vasos cuando ese no existe. Con laparoscopía se podría realizar este mismo trabajo, siendo menos invasiva.

La ventaja del procedimiento quirúrgico abierto reside en la incisión inguinal, con extensión intraabdominal cuando es necesario, pues permite localizar y abordar los testículos de diferente ubicación, realizando orquiectomía cuando es preciso o descendiendo con orquidopexia estándar, en lo posible sin sección de los vasos espermáticos, los restantes.

\section{Referencias}

1. Scorer $\mathrm{CG}$ and Farrington GH: Congenital detormities of the testis and epididimys. New York: Appleton - century - crafts, 1971: 56.

2. Redmes JF. Inpalpable testis; observation based on 208 consecurives operations for undescended testes. 1 Urol 1980; $124: 379$.

3. Cunpleh $H E$ : incidence of nalignant growth of the undescended testicle. Arch Surg 1942; 44: 353.

4. Snolko MJ, Kuplan EW. Brich WA: Location and fate of the nonpalpable testis in children. J Urol 1983: 129; 1204.

5. Wif $R$. Exner GU. Koffmann $F$. et al: Treatment of cryptorchidism by intranasal synthetic LHRH. Lancet 1977: $2: 518$.

6. Jomes PG: Undescended testis. Aust Paed J 1966: 2 : 36 .

7. Tibbs DJ: Unilateral absence of the testis. Brit J Surg 1961: $48: 601$

8. Levift $S B, K o g(m) S J$. Engel RM, et al: The impalpable testis: a ralional approach to management. J Urol 1978: 120: 515.

9. Cortesi N. Ferrari P. Zanberde E. et at: Diagnosis of bilateral aboominal cryptorchidisin by laparoscopy. Endoscopy 1976; 8: 33 .

10. Bicm DA: Two steps orchiopexy with pelviscopic clip ligation of the espermalic vassels. J Urol 199I; 145: 1030 
11. Castilho LN. Ferreira V: Lapatoscopic pediatric orcbiectomy J Endourol 1992; 6: 153.

12. Thomas MD. Mercar LC: Laparoseopic orchiectomy for unilateral intrabdominal testis. I Urol 1992: I48: [25].

13. Jordan $G$. Winstow $B$ : Laparoscopy single stage and staged orchiopexy. J Urol 1994; 152:1249-1252.

14. Caldumone A, Amaral J: Laparoscopy stage 2 FowlerStephens orchiopexy. J Urol 1994; 152: 1253-1256.

15. Moore $G G$. Peters $C A$, Baver $S B$, et al.: Laparoscopic evaluation of the non-palpable tesis. I Urol 1994; $151: 728$.
16. Elder $J:$ Laparoscopy for impalpable lestis: significance of the process vaginalis. J Urol 1994: 1.52 : 776.

17. Rednem JF: Empalpable testis. J Lrol 1980: 124: 379.

18. Smolko MJ, Kaplan GW, Broch WA: Location and late of the nonpalpable testis in the children. J Urol 1983: 129: 1204

19. Guiney EJ. Corbaly $M$, Malone $P$ : Laparoscopy and the management of the impalpable testi. $\mathrm{Br} J$ Utol 1989: 63:313.

20. Gibbons MP, Cronne JC, Ducheft JW: Management of the abdominal undesceded lestis. J Urol 1979: 122: 76.

\section{AVISO A LOS AUTORES}

Por acuerdo del Comité Editorial, la Revista Chilena de Pediatría devolverá sin tramitar todos los trabajos que no den estricto cumplimiento al Reglamento de Publicaciones y a las Instrucciones de los Autores que se editan en cada número de la revista. 